\title{
Roche lobe effects on expanded upper atmospheres of short-periodic giant exoplanets
}

\author{
G. F. Jaritz ${ }^{1,2}$, S. Endler ${ }^{2,3}$, D. Langmayr ${ }^{2}$, H. Lammer ${ }^{2}$, J.-M. Grießmeier ${ }^{4}$, \\ N. V. Erkaev ${ }^{5}$, and H. K. Biernat ${ }^{1,2,3}$ \\ ${ }^{1}$ Institute for Geophysics, Astrophysics, and Meteorology, University of Graz, Universitätsplatz 5, 8010 Graz, Austria \\ e-mail: gerald.jaritz@stud.uni-graz.at \\ 2 Space Research Institute, Austrian Academy of Sciences, Schmiedlstrasse 6, 8042 Graz, Austria \\ e-mail: [helfried.biernat; helmut.lammer; daniel.langmayr]@oeaw.ac.at \\ ${ }^{3}$ Institute for Theoretical Physics, University of Graz, Universitätsplatz 5, 8010 Graz, Austria \\ e-mail: stefan.endler@stud.uni-graz.at \\ ${ }^{4}$ Institut für Theoretische Physik, Technische Universität Braunschweig, Mendelssohnstrasse 3, 38106 Braunschweig, \\ Germany \\ e-mail: j-m.griessmeier@tu-bs.de \\ 5 Institute for Computational Modelling, Russian Academy of Sciences, Krasnoyarsk 36, Russian Federation \\ e-mail: erkaev@icm.krasn.ru
}

Received 13 December 2004 / Accepted 2 May 2005

\begin{abstract}
Theoretical studies and recent observational evidence of the expansion of the atmospheres of short-periodic exoplanets show that the atmospheres extend up to several planetary radii. This indicates that the atmospheres experience blow-off conditions. Because of the short orbital distance to their host stars, the expansion of the upper atmosphere is no longer radially symmetric, but depends on the direction to the central body, resulting in a deformation of the expanded atmosphere. We show the connection between atmospheric expansion, tidal forces and effects of the Roche potential and find that HD $209458 \mathrm{~b}$, OGLE-TR-10 $\mathrm{b}$ and OGLE-TR-111 $\mathrm{b}$ are most likely in a state of classical hydrodynamical blow-off, because the distance where blow-off can occur is less than the distance to the Lagrangian point L1. On the other hand, OGLE-TR-56 b, OGLE-TR-113 b, OGLE-TR-132 b and TreS-1 experience a geometrical blow-off defined by the Roche lobe as proposed by Lecavelier des Etangs et al. (2004, A\&A, 418, L1). Our results have important implications for the evolution of short periodic gas giants, because the Roche lobe overflow of the atmosphere can lead to lower mass loss rates over the exoplanets history, compared to gas giants which experience hydrodynamic expansion and loss unaffected by this boundary. Thus, massive exoplanets like OGLE-TR-56 b in very close orbital distances are subject to geometrical blow-off conditions, this results in a total mass loss for this particular exoplanet of the order of about $3 \times 10^{-2} M_{\mathrm{pl}}$ over the planets age, even if current mass loss rates of about $2 \times 10^{11} \mathrm{~g} \mathrm{~s}^{-1}$ are calculated. If the exoplanet effected by the geometrical blow-off is more massive, the mass loss rate is even lower. However, giant exoplanets like HD 209458 b, OGLE-TR-10 b and OGLE-TR-111 b at orbital distances of about 0.05 AU may experience classical hydrodynamic blow-off conditions, which can result in higher mass loss rates. Thus, such planets may shrink to their core sizes during the X-ray and EUV active periods of their host stars as proposed by Lammer et al. (2003, ApJ, L121, 598) and Baraffe et al. (2004, A\&A, 419, L13).
\end{abstract}

Key words. radiation mechanisms: non-thermal - acceleration of particles - plasmas - turbulence - gamma rays: theory methods: analytical

\section{Introduction}

Tidal forces and mass transfer processes from short-periodic gas giants to their host stars should act at $\approx 20 \%$ of the presently known $>150$ exoplanets (status: June 2005), which have orbital distances $<0.1 \mathrm{AU}$, or orbital periods $<11$ days.

Since the observation of an extended upper atmosphere around HD 209458 b by Vidal Madjar et al. (2003), different blow-off scenarios for evaporating hydrogen-rich atmospheres are discussed in the literature (Sasselov 2003; Lammer et al. 2003; Lecavelier des Etangs et al. 2004; Yelle 2004; Vidal Madjar et al. 2004; Grießmeier et al. 2004; Baraffe et al. 2004; Erkaev et al. 2005; Tian et al. 2005).

It is shown by Lammer et al. (2003) and in more detail by Yelle (2004) that exospheres of hydrogen-rich giant exoplanets in orbits with semi-major axes from $0.01-0.1 \mathrm{AU}$ can be heated to temperatures of up to $10000-20000 \mathrm{~K}$ by X-rays and 
extreme ultraviolet radiation (XUV) from the central star. The lower thermosphere is cooled primarily by radiative emissions from $\mathrm{H}_{3}^{+}$, created by photo-ionization of $\mathrm{H}_{2}$ and subsequent ion chemistry (Yelle 2004). These high temperatures cause a large escape flux of the atmospheric gas, implying that the upper atmosphere is cooled primarily by atmospheric expansion and high loss rates (Lammer et al. 2003; Yelle 2004; Tian et al. 2005).

With the Space Telescope Imaging Spectrograph (STIS) onboard of the Hubble Space Telescope (HST), Vidal Madjar et al. (2003) determined a lower limit of the atmospheric loss rate from HD $209458 \mathrm{~b}$ of $\geq 10^{10} \mathrm{~g} \mathrm{~s}^{-1}$ by Lyman- $\alpha$ absorption features. Assuming classical hydrodynamic conditions, Lammer et al. (2003) calculated the maximum possible energylimited hydrogen loss rate of HD $209458 \mathrm{~b}$ corresponding to the present age of its host star $(\approx 5.2 \mathrm{Gyr})$ to be $\approx 10^{12} \mathrm{~g} \mathrm{~s}^{-1}$.

However, Lecavelier des Etangs et al. (2004) proposed an alternative scenario for short-periodic exoplanets, which they call geometrical blow-off, by arguing that due to the high temperatures the exobase level can reach the Roche lobe before classical hydrodynamic conditions may develop. The Roche lobe is defined as the last equipotential around a planet. Beyond this, the surfaces of constant potential encompass the host star. Because particles beyond the Roche lobe can definitely escape, Lecavelier des Etangs et al. (2004) argued that the Roche lobe can be seen as an equivalent to the exobase level. However, depending on planetary and stellar masses, planetary sizes, stellar types and orbital distances from the host stars both scenarios, the classical hydrodynamic blow-off, or the geometrical blow-off may occur.

The aim of this paper is to investigate in more detail the implications of both cases. In Sect. 2 we discuss the theory of the Roche potential between short-periodic gas giants and their host stars. In Sect. 3 we compare the Roche lobe distance with calculated atmospheric expansion radii of seven short-periodic giant exoplanets with known mass and radius, and discuss the consequences for the atmospheric evolution.

\section{Roche potentials of close-in exoplanet-stellar environments}

By studying the three-dimensional Roche potential of shortperiodic gas giants, one takes into account the fact that the atmosphere may be gravitationally distorted. By assuming two point masses $M_{1}$ and $M_{2}$, separated by a distance $a$ and in circular orbits around their common center of mass, the energy per unit mass of a test particle inside the system can be derived from the potential (Paczyński 1971)

$\Phi=-\frac{G M_{1}}{r_{1}}-\frac{G M_{2}}{r_{2}}-\frac{G\left(M_{1}+M_{2}\right) s^{2}}{2 a^{3}}$,

where $s$ is the distance of the particle from the binary's center of mass, and $r_{1}$ and $r_{2}$ stand for the particle's distance to the star and planet, respectively. $G$ is Newton's gravitational constant. Surfaces on which $\Phi$ is constant correspond to surfaces of constant potential energy, the Roche potentials.

By introducing the mass ratio $\delta=M_{\mathrm{pl}} / M_{\star}$, between the exoplanet's mass and its host star and by normalizing Eq. (1) one can write the potential $\Phi$ in a $3-D$ coordinate system as

$$
\begin{aligned}
\Phi(x, y, z)= & -\frac{1}{\sqrt{x^{2}+y^{2}+z^{2}}}-\frac{\delta}{\sqrt{(x-1)^{2}+y^{2}+z^{2}}} \\
& -\frac{1}{2}(1+\delta)\left[\left(x-\frac{\delta}{1+\delta}\right)^{2}+y^{2}\right] .
\end{aligned}
$$

In Eq. (2), the first term represents the potential of the primary star and the second the potential of the exoplanet. The third term is a result of the orbital motion of the system and includes the mass ratio. $x-y$ is the orbital plane and $z$ is perpendicular to it. For illustration of the Roche potentials we calculate the potentials for a planetary system with a mass ratio of 0.013 . The Roche potentials are shown with normalized parameters in Fig. 1.

For this potential, there are two locations, the Lagrangian point L1 and the Lagrangian point L2 which are saddle points. Both points lie on the line which joins the planet and the host star, where L1 is located between them and L2 is farther away from the star than the planet. The planetocentric distances of L1 and $\mathrm{L} 2$ are (Gu et al. 2003)

$r_{\mathrm{L} 1, \mathrm{~L} 2}=\left[\beta \mp \frac{\beta^{2}}{3}\right] a$,

with

$\beta=\left(\frac{\delta}{3}\right)^{\frac{1}{3}}$

The Roche potential has its largest planetocentric distance, which is related to the last equipotential surface for which a particle can be bound to the planet ( $\mathrm{Gu}$ et al. 2003). At the distance of the Lagrangian point $r_{\mathrm{L} 1}$ an expanding atmosphere cause a flux of gas towards the star, while the gas which expands beyond $r_{\mathrm{L} 2}$ spirals outwards to form an excretion disk. The Roche potential has its largest planetocentric distance, which is related to the last equipotential surface for which a particle can be bound to the planet $(\mathrm{Gu}$ et al. 2003). Furthermore, it is important to note that the equipotential surfaces containing the L1 and L2 points are distorted from spherical symmetry.

\section{Implication of the Roche potential for atmospheric blow-off}

For studying the effect of the Roche potential on expanded atmospheres of hot gas giants we have chosen HD $209458 \mathrm{~b}$ (Mazeh 2000; Charbonneau et al. 2000; Henry et al. 2000; Barman et al. 2002; Vidal Madjar et al. 2003), OGLE-TR-10 b (Bouchy et al. 2005; Konacki et al. 2005), OGLE-TR-56 b (Konacki et al. 2003; Burrows et al. 2004; Baraffe et al. 2004; Torres et al. 2004), OGLE-TR-111 b (Pont et al. 2004), OGLE-TR-113 b (Bouchy et al. 2004; Konacki et al. 2004), OGLE-TR-132 b (Bouchy et al. 2004; Moutou et al. 2004), and TreS-1 (Alonso et al. 2004) where the mass and radius are known. The parameters of these exoplanets and their host stars, as well as $r_{\mathrm{L} 1}$ and their atmospheric expansion radii $r_{\exp }$ calculated for classical hydrodynamic conditions by using the 


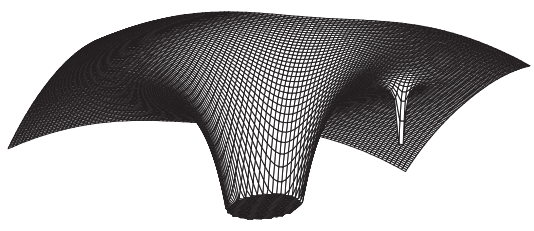

a

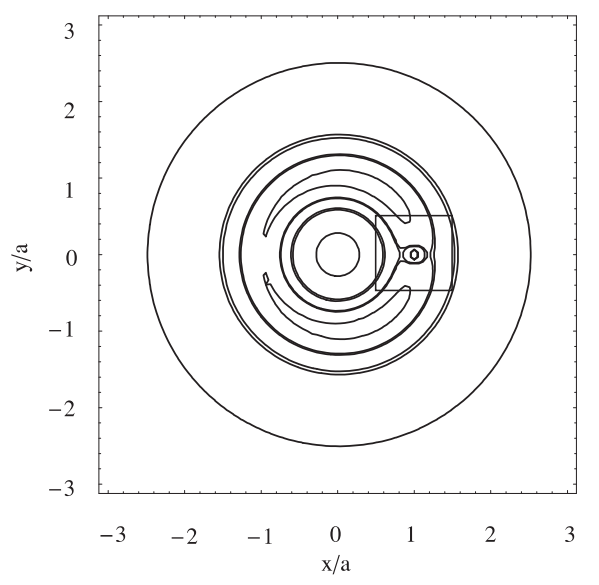

$\mathrm{b}$

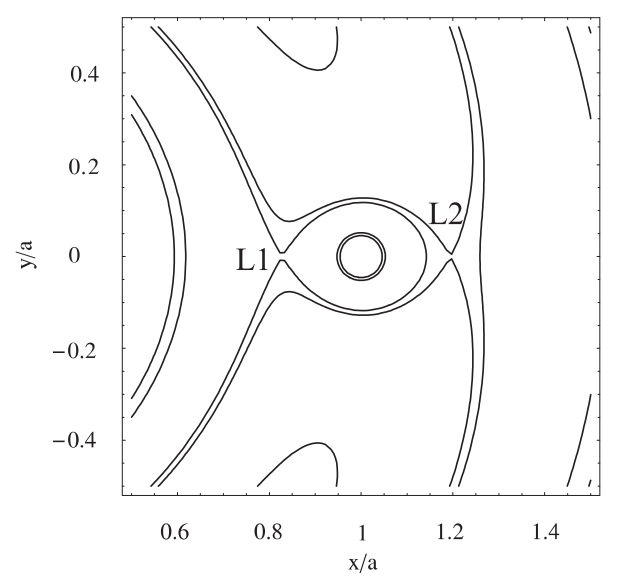

c

Fig. 1. For illustration of the Roche potentials we calculate the potentials for a planetary system with a mass ratio of 0.013 a) in three dimensions and b) as contours. The square in $\mathbf{b}$ ) is blown up in $\mathbf{c}$ ). The two peaks in a) show the potential close to the star and planet, respectively.

equations of Watson et al. (1981) and Lammer et al. (2003) are shown in Table 1.

The effective temperatures used in our calculations for HD $209458 \mathrm{~b}$ and OGLE-TR-56 b are taken from Barman et al. (2002), Sasselov (2003) and Konacki et al. (2005). For the other exoplanets in our study we calculated $T_{\text {eff }}$ with the following formula:

$T_{\text {eff }}=\left(\frac{3}{4} \tau+\frac{1}{2}\right)^{\frac{1}{4}} T_{\text {star }}\left(\frac{1-A}{4}\right)^{\frac{1}{4}}\left(\frac{R_{\text {star }}}{d}\right)^{\frac{1}{2}}$,

where $\tau$ is the optical depth, $T_{\text {star }}$ is the effective temperature of the star, $A$ the albedo of the planet, $R_{\text {star }}$ stands for the radius of the star, and $d$ is the distance to the star in AU. It

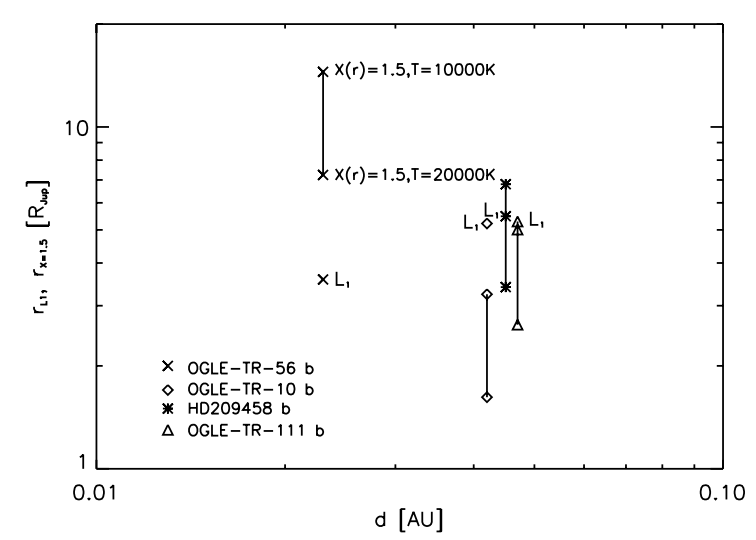

Fig. 2. Relevant radii $r_{\mathrm{X}=1.5}$ and $r_{\mathrm{L} 1}$ for OGLE-TR-56 b, OGLE-TR-10 b, OGLE-TR-111 b and HD 209458 b. For OGLE-TR-56 b, $r_{\mathrm{L} 1}$ is clearly less than $r_{\mathrm{X}=1.5}$, which indicates that the exoplanet is most likely in a stage of geometric blow-off, while the three other exoplanets are most likely in a stage of a classical hydrodynamic blow-off regime.

is based on the Stefan-Boltzmann Law, which describes the radiation of a black body. Since exoplanetary atmospheres are no ideal black bodies we use Eddington's approximation (Böhm-Vitense 1989) to calculate $T_{\text {eff }}$ at a certain optical depth $\tau$ for a grey atmosphere. For the albedo of the planet, we use Jupiter's value $A=0.52$.

Lecavelier des Etangs et al. (2004) pointed out that at temperatures of $\approx 10000 \mathrm{~K}$, the exobase of HD $209458 \mathrm{~b}$ merges with the Roche lobe, which they estimate to be $r_{\text {eff }} \approx 3.6 r_{\text {Jup }}$ $\left(2.67 r_{\mathrm{pl}}\right)$. This effective radius $r_{\mathrm{eff}}$, however, corresponds to a spherical approximation of the Roche lobe (Eggleton 1983), which is generally used for binary stellar systems, where small distance variations are negligible. The approximated radius $r_{\mathrm{eff}}$ always yields results smaller than $r_{\mathrm{L} 1}$. Note that the shape of the potential will guarantee that the Roche lobe is reached everywhere at the same time as in L1. This is the reason why the radius $r_{\exp }{ }^{1}$ of an expanded exoplanetary atmosphere should better be compared to $r_{\mathrm{L} 1}$ (Gu et al. 2003) rather than to the spherically averaged $r_{\text {eff }}$ (Eggleton 1983).

Classical hydrodynamical blow-off occurs at the altitude level $r_{X=1.5}$, where the thermal escape parameter $X=$ $(G M m) /(k T r)$ reaches values of $\approx 1.5$. With the planetary parameters of HD $209458 \mathrm{~b}$ and temperatures of $\approx 15000 \mathrm{~K}$, this altitude level is at $r_{X=1.5}=6.61 r_{\mathrm{Jup}}\left(4.9 r_{\mathrm{pl}}\right)$, which is beyond $r_{\mathrm{L} 1}$ of $\approx 5.482 r_{\mathrm{Jup}}\left(4.06 r_{\mathrm{pl}}\right)$. For temperatures of $\approx 18000-20000 \mathrm{~K}$, classical hydrodynamic blow-off occurs close to $r_{\mathrm{L} 1}$ or even at lower altitudes of $r_{X=1.5} \approx 4.85 r_{\mathrm{Jup}}$ $\left(3.6 r_{\mathrm{pl}}\right)$, respectively. For these temperatures, one finds $r_{\mathrm{eff}}<$ $r_{\exp }<r_{X=1.5}<r_{\mathrm{L} 1}$ and the majority of the XUV flux is deposited below $r_{\mathrm{L} 1}$, which results in classical hydrodynamic blow-off conditions, not influenced by the Roche lobe.

As shown in Fig. 2, by using temperatures of $10000 \mathrm{~K}$ and $20000 \mathrm{~K}$ we find that the escape parameter reaches 1.5 at altitudes of about 3.24 and $1.62 r_{\text {Jup }}$ for OGLE-TR-10 b and at altitudes of about 5 and $2.65 r_{\text {Jup }}$ for OGLE-111 b,

1 The expansion radius $r_{\exp }$ is the distance, where the majority of the stellar XUV radiation is absorbed by the atmospheric gas. 
Table 1. Relevant parameters of HD 209458 b (Barman et al. 2002; Vidal Madjar et al. 2003), OGLE-TR-56 b (Burrows et al. 2004; Baraffe et al. 2004), OGLE-TR-132 b (Moutou et al. 2004), OGLE-TR-113 b (Bouchy et al. 2004), OGLE-TR-111 b (Pont et al. 2004), OGLE-TR-132 b (Bouchy et al. 2004; Moutou et al. 2004), OGLE-TR-10 b (Bouchy et al. 2005; Konacki et al. 2005) and TreS-1 (Alonso et al. 2004) for the calculation of the atmospheric expansion radius $r_{\exp }$ and for the calculation of $r_{\mathrm{L} 1}$.

\begin{tabular}{cccccccc}
\hline \hline Exo-system (Star type) & $M_{\star}\left[M_{\odot}\right]$ & $M_{\mathrm{pl}}\left[M_{\mathrm{Jup}}\right]$ & $r_{\mathrm{pl}}\left[r_{\mathrm{Jup}}\right]$ & $a[\mathrm{AU}]$ & $T_{\mathrm{eff}}[\mathrm{K}]$ & $r_{\mathrm{exp}}\left[r_{\text {Jup }}\right]$ & $r_{\mathrm{L} 1}\left[r_{\text {Jup }}\right]$ \\
\hline HD 209458 b (G0V) & 1.05 & 0.69 & 1.43 & 0.045 & 1800 & 2.97 & 5.48 \\
OGLE-TR-56 b (G) & 1.04 & 1.45 & 1.23 & 0.023 & 1900 & 5.89 & 3.58 \\
OGLE-TR-132 b (F) & 1.34 & 1.01 & 1.15 & 0.031 & 1750 & 4.46 & 3.95 \\
OGLE-TR-113 b (K) & 0.77 & 1.35 & 1.08 & 0.023 & 1000 & 10.42 & 3.85 \\
TreS-1 (K0V) & 0.87 & 0.75 & 1.08 & 0.039 & 1000 & 5.79 & 4.75 \\
OGLE-TR-111 b (G or K) & 0.82 & 0.53 & 1.00 & 0.047 & 1070 & 3.83 & 5.01 \\
OGLE-TR-10 b (G or K) & 1.22 & 0.57 & 1.24 & 0.042 & 1140 & 3.87 & 5.22 \\
\hline
\end{tabular}

respectively. Because, the distance of $r_{\mathrm{L} 1}$ is at about 5 and $5.2 r_{\text {Jup }}$ for OGLE-TR-10 $\mathrm{b}$ and OGLE-TR-111 b, these exoplanets are most likely also in a state of classical hydrodynamic blow-off.

As one can see in Table 1, for OGLE-TR-56 b, OGLETR-113 $b$ and OGLE-TR-132 b and TreS-1 b, the relevant distances $r_{\exp }$ and $r_{X=1.5}$ (which is $>r_{\exp }$ ) are much larger than $r_{\mathrm{L} 1}$. Because, the radius $r_{X=1.5}$ where an exosphere changes to hydrodynamic conditions is $>r_{\mathrm{L} 1}$, OGLE-TR-56 $\mathrm{b}$ (see Fig. 2), OGLE-TR-113 b, OGLE-TR-132 b and TreS-1 b should experience geometrical blow-off conditions as proposed for HD 209458 b by Lecavelier des Etangs et al. (2004).

Note that for the estimation of $r_{\exp }$, the stellar XUV flux is required. Because the XUV fluxes of the host stars for OGLE-TR-113 b (K star), OGLE-TR-132 b (F star) and TreS-1 b (K0V star) are not known, we use the XUV flux of the present Sun (G-star), scaled to the orbital distance of the exoplanet. However, we suggest that different XUV fluxes related to the real spectral types of these host stars may not change the fact that all three planets experience geometrical blow-off.

One should also note that the radiation pressure of the host star can shift the gas below L1 towards the direction of the second Lagrangian point L2 where the planetary wind may create a cometary-like tail of the evaporating gas.

As described above, HD 209458 b may experience classical hydrodynamic blow-off if the temperature of the upper atmosphere reaches the critical value before the exobase level moves beyond $r_{\mathrm{L} 1}$. This suggestion is consistent with recent observations of four transits of HD $209458 \mathrm{~b}$ with the STIS spectrograph on board HST by Vidal Madjar et al. (2004). During these transits oxygen and hydrogen were observed in the extended upper atmosphere of HD $209458 \mathrm{~b}$ and up to the Roche lobe. Because of that, the thermal escape parameter $X$ of $\mathrm{O}$ and $\mathrm{C}$ at $r_{\mathrm{L} 1}$ for $10000 \mathrm{~K}$ considered by Lecavelier des Etangs et al. (2004) are $\approx 60$ and 44. Both species will not diffuse in the kinetic regime upward to the planetocentric distances where they were observed.

Figure 3 shows a 3-D simulation of part of the 3.52 day orbit period of HD $209458 \mathrm{~b}$ and its expanded atmosphere which is deformed due to tidal forces. The host star is shown as a point-mass and the red line represents the direction of the orbital period.

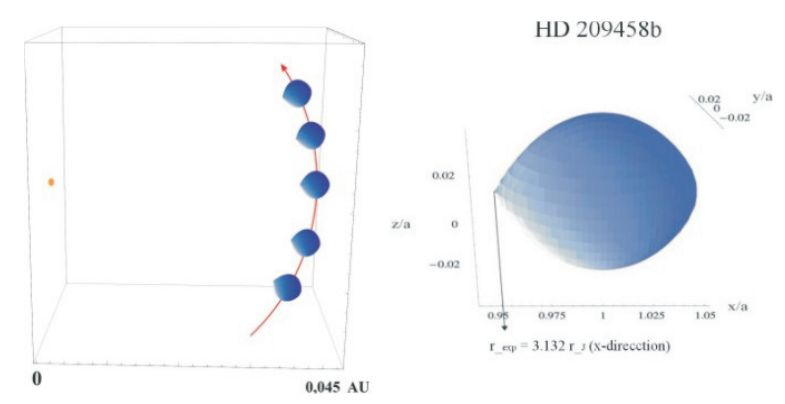

Fig. 3. Left panel: expanded atmosphere of HD 209458 b under the influence of the tidal forces of its host star, which is pictured as a point-mass. The size of the planet is not in scale. Right panel: detailed view of the normalized expanded atmosphere of HD 209458 b under the influence of tidal forces. Note that the tidal deformation leads to an atmospheric radius which slightly deviates form the more idealized expansion radius $r_{\text {exp }}$.

The results of our study have important implications for evolutionary aspects of planetary mass loss. If we apply a hydrodynamical model and a upper atmospheric boundary at $r_{\mathrm{L} 1}$ corresponding to about $3.58 r_{\text {Jup }}$ and temperatures of about $15000 \mathrm{~K}$ for OGLE-TR-56 b, we obtain atomic hydrogen loss rates at the Roche Lobe overflow of about $\leq 2 \times 10^{11} \mathrm{~g} \mathrm{~s}^{-1}$ for the present age of the system of $3 \pm 1$ Gyr (Sasselov 2003). These loss rates are several orders of magnitude higher than estimated by Sasselov (2003), who calculated the Jeans loss by using the effective temperature $T_{\text {eff }}$ of $1900 \mathrm{~K}$, but 25 times lower than the energy-limited mass loss for OGLE-TR-56 $b$ calculated by Lammer et al. (2003). By integrating these loss rates over the age of the planetary system and by considering a 100 times higher XUV flux at the early periods (Lammer et al. 2003; Ribas et al. 2005) we obtain a maximum mass loss for OGLE-TR-56 b of about $3.5 \times 10^{-2} M_{\mathrm{pl}}$. This total mass loss is lesser than the calculated XUV-driven energy-limited loss as shown in Lammer et al. (2003, Fig. 3b) where the Roche lobe cut off was not taken into account.

Our results imply that Jovian-type exoplanets affected by Roche lobe-limited loss may be less effected on mass loss over their lifetimes compared to hot gas giants, which experience classical hydrodynamical blow off. For planets like HD 209458 b, OGLE-TR-10 b and OGLE-TR-111 b, which most likely experience classical hydrodynamic blow-off, strong 
mass loss may be possible as calculated for HD 209458 b by Lammer et al. (2003, Fig. 3a) and Baraffe et al. (2004). As we have shown in our study, the influence of the Roche lobe to an expanded atmosphere is very sensitive to the parameters of the host star and the planet itself, therefore, one can not generalize the calculated mass loss rates for all exoplanets with short orbital periods.

The evaporation studies of Baraffe et al. (2004) lead to the existence of a critical planetary mass $m_{\text {crit }}$ which depends on the orbital distance. Below this, the evaporation timescale becomes shorter than the thermal timescale of the exoplanet. For planets with initial masses below $m_{\text {crit }}$, strong hydrodynamic evaporation during XUV active stellar periods can lead to a rapid expansion of the outer planetary layers and of the total planetary radius $r_{\mathrm{pl}}$, speeding up the evaporation process. Consequently, they found that the exoplanet does not survive as long as estimated by a simple application of mass loss rates without following consistently its evolution. Baraffe et al. (2004) speculate also that HD 209458 b might be in such a dramatic phase. Future studies, which are beyond the scope of this paper, should similarly investigate the mass-radius evolution for OGLE-TR-10 b and OGLE-TR-111 b, which experience most likely classical hydrodynamic blow off and have also larger planetary radii compared to the massive OGLE-TR-56 b, which is found to be in geometrical blow-off.

\section{Conclusions}

A detailed investigation of Roche lobe effects to the expanded atmospheres of seven short-periodic giant exoplanets indicate that the atmospheres of HD $209458 \mathrm{~b}$, OGLE-TR-10 b and OGLE-TR-111 b are most likely in a state of classical hydrodynamical blow-off, because the critical level where blow-off occurs can be reached before the exobase level reaches the Lagrangian point $r_{\mathrm{L} 1}$. In the cases of OGLE-TR-56 b, OGLE-TR-113 b, OGLE-TR-132 $b$ and TreS- $1 b$ the critical level for classical hydrodynamic blow-off cannot be reached. These exoplanets experience geometrical blow-off as proposed by Lecavelier des Etangs et al. (2004) resulting in less efficient atmospheric loss rates over their lifetimes. However, the results of our study can be investigated by the statistical analysis of CoRoT planet detections in the near future, because it indicates that we may have two populations of short periodic giant exoplanets, which depends on stellar and planetary parameters: One population which is strongly effected by a Roche lobe cut off resulting in less mass loss over evolutionary periods and a second population, which experiences classical hydrodynamical blow off, which may evaporate planets to their core sizes.

Acknowledgements. H. Lammer and H. K. Biernat thank the Austrian Academy of Sciences "Verwaltungsstelle für Auslandsbeziehungen". This work is supported by the INTAS-ESA Project 99-01277, by the Austrian "Fonds zur Förderung der wissenschaftlichen Forschung" (FWF) under project P17100-N08, by grants No. 01-05-02003 and
No. 01-05-65070 from the Russian Foundation of Basic Research, and by project No. I.2/04 from Österreichischer Austauschdienst (ÖAD). The authors also thank the Austrian Ministry for Science, Education and Culture (bm:bwk) and ASA for funding the CoRoT project. Finally, the authors thank an anonymous referee for constructive comments, which helped to improve the content of the paper. This study was supported by the International Space Science Institute (ISSI) and carried out in the frame of the ISSI Team "Formation, Structure and Evolution of Giant Planets".

\section{References}

Alonso, R., Brown, T. M., Torres, G., et al. 2004, ApJ, 613, 153

Baraffe, I., Selsis, F., Chabrier, G., et al. 2004, A\&A, 419, L13

Barman, T. S., Hauschildt, P. H., Schweitzer, A., et al. 2002, ApJ, 569, L51

Böhm-Vitense, E. 1989, Introduction to stellar astrophysics, Vol. 1 Basic stellar observations and data, Vol. 2 - Stellar atmospheres (Cambridge, New York: Cambridge University Press)

Burrows, A., Hubeny, I., Hubbard, W., Sudarsky, D., \& Fortney, J. 2004, ApJ, 610, L53

Bouchy, F., Pont F., Santos N., Melo C., Mayor M., Queloz D. \& Udry S., 2004, A\&A, 421, L13

Bouchy, F., Pont, F., Melo, C., et al. 2005, A\&A, 431, 1105

Charbonneau, D., Brown, T., Latham, D., \& Mayor, M. 2000, ApJ, 529, L45

Eggleton, P. P. 2003, ApJ, 268, 368

Erkaev, N. V., Penz, T., Lammer, H., et al. 2005, ApJS, in press

Grießmeier, J.-M., Stadelmann, A., Penz, T., et al. 2004, A\&A, 425, 753

Gu, P.-G., Lin, D. N. C., \& Bodenheimer, P. H. 2003, ApJ, 588, 509

Henry, G. W., Marcy, G. W., Butler, R. P., \& Vogt, S. S. 2000, ApJ, 529, L41

Konacki, M., Torres, G., Jha, S., \& Sasselov, D. D. 2003, Nature, 421, 507

Konacki, M., Torres, G., Sasselov, D., et al. 2004, ApJ, 609, L37

Konacki, M., Torres, G., Sasselov, D., \& Jha, S. 2005, ApJ, submitted [arXiv: astro-ph/0412400]

Lammer, H., Selsis, F., Ribas, I., et al. 2003, ApJ, L121, 598

Lecavelier des Etangs, A., Vidal-Madjar, A., McConnell, J. C., \& Hébrard, G. 2004, A\&A, 418, L1

Mazeh, T., Naef, D., Torres, G., et al. 2000, ApJ, 532, L55

Moutou, C., Coustenis, A., Schneider, J., et al. 2001, A\&A, 371, 260

Moutou, C., Pont, F., Bouchy, F., \& Mayor, M. 2004, A\&A, 424, L31

Paczyński, B. 1971, ARA\&A, 9, 183

Pont, F., Bouchy, F., Queloz, D., et al. 2004, A\&A, 426, L15

Ribas, I., Guinan, E. F., Güdel, M., \& Audard, M. 2005, ApJ, 622, 680

Sasselov, D. 2003, ApJ, 596, 1327

Shkolnik, E., Walker, G., \& Bohlender, D. 2003, ApJ, 597, 1092

Tian, F., Toon, O. B., Pavlov, A. A., \& Sterck, H. De. 2005, ApJ, 621, 1049

Torres, G., Konacki, M., Sasselov, D., \& Jha, S. 2004, ApJ, 609, 1071

Vidal Madjar, A., Lecavelier des Etangs, A., Desert, J.-M., et al. 2003, Nature, 422, 143

Vidal Madjar, A., Lecavelier des Etangs, A., Desert, J.-M., et al. 2004, ApJ, in press

Watson, A. J., Donahue, T. M., \& Walker, J. C. G. 1981, Icarus, 48, 150

Yelle, R. V. 2004, Icarus, 170, 167 